\title{
ODEs model of foreign body reaction around peripheral nerve implanted electrode
}

\author{
G. Di Pino, D. Formica, L. Lonini, D. Accoto, A. Benvenuto, S.Micera Senior Member IEEE, P.M. \\ Rossini and E. Guglielmelli, Member IEEE.
}

\begin{abstract}
The foreign body reaction that the neural tissue develops around an implanted electrode contributes to insulate the probe and enhances the electrical and mechanical mismatch. It is a complex interaction among cells and soluble mediators and the knowledge of this phenomenon can benefits of formal and analytical methods that characterize the mathematical models. This work offers a lumped component model, described by ordinary differential equations, that taking into account the main geometrical (size, shape, insertion angle) and chemical (coating surface) properties of the implant predict the thickness of the fibrotic capsule in a time frame when the reaction stabilizes. This tool allows to evaluate different hypothetical solutions for accounting the tissue-electrode mismatch.
\end{abstract}

\section{INTRODUCTION}

E lectrodes show an acceptable capability of recording high quality neural signals and of properly stimulating for sensorial feedback delivering, but what more deserves the attention of neural interfacing technology designers is the long term endurance of performance that is still not acceptable for human applications. Foreign body reaction (FBR) against electrode is part of a general response of the nervous system to implanted material. It affects both the short and even more the long-term performance of the device [1, 2], in registration and in stimulation cues and it is strongly influenced by the electrode-tissue interface. Improving comprehension about this response of the body could give new insights in the development of more tissue-integrated devices. Inflammatory reactions, and all the sub-processes that are involved in it, are an optimal example of complex processes, with multiple interactions among several cells and soluble mediators, that evolve in time. Their study can benefit from the appliance of formal analytical methods typical of the mathematical models [3]. Although the recognized importance of FBR in determining the performance of electrodes and other implanted devices (i.e. orthopedic prosthesis and insulin dispenser), and its inclination to be modeled, literature is poor of FBR mathematical models. Are available only an old work by Nichols and colleagues about FBR against aspecific material [4], that is not very useful being missed all the used parameters, and a technical report that faces in particular the FBR to neural implants that introduce the argument, but remands to a future, not yet published, scientific article [5]. Here a simple model, taking into account the main geometrical (size, shape, insertion angle) and chemical (coating surface) properties of the implanted electrode has been developed, in order to evaluate the thickness of the fibrotic capsule in a time frame when the reaction stabilizes (about 4 weeks), while keeping low the computational cost.

\section{METHODS}

\section{A. Blocks schemes and specifications of the model}

To realize the model we adopt a lumped component model, described by ordinary differential equations (ODEs model), that assumes some theoretical main simplifications:

- peripheral nervous tissue that receives the implant is considered homogeneous;

- in the set of points, laying on a section plane perpendicular to the longitudinal axis of the electrode, that are equally distant from the electrode the evolution of the FBR is equal;

- blood and tissues adjacent to the insertion site (here defined as region D and C) are an unlimited reservoir of cells compared to the amount recruited in the FBR.

The model presents an initial on/off stimulus, corresponding to the electrode insertion, that start the inflammatory process through the mechanical damage produced at the insertion site. Variation in size, shape and insertion angle of the electrode modulates the amount of the damage, while, given a particular damage, the biochemistry of the surface coating modulates the further biological process producing the inflammatory reaction. Once this reaction is started, a balance among the reinforcement and the resolution of the inflammation is mainly due to the counter effects of pro and anti-inflammatory cytokines (PIC and AIC). The fibrotic evolution is responsible for the stabilization of the electrode encapsulation. Secondary micromovements of the electrode that act after the insertion push toward an increase and a chronicity of the inflammation.

The strategy followed in the model needs to define four spatial regions where the variables are homogeneous. Going from the electrode surface to the periphery, region $\mathrm{A}$ is the tissue-electrode interface occupied by Foreign Body Giant Cells (FBGC), region $\mathrm{B}$ the peri-electrode tissue made by Extra-Cellular Matrix (ECM), region $\mathrm{C}$ is the ensemble of tissues adjacent to the implant and region $\mathrm{D}$ represent the blood volume inside the peri-implant vessels. The electrode is in contact only with region A. Region A and B are concentric and together form the granulation tissue of the capsule of which the model proposes to give the thickness as its output. Region B is in contact with both region D through the blood vessel wall barrier and with Region C. In region A temporal evolution of inflammation sees the immediate adsorption of proteins on the electrode surface that work as adaptor for the proximal monocytes adhesion followed by the activation in macrophages cell-type and the fusion to form FBGC. In region B the insertion damage produce the blood vessels rupture and the consequent formation of a blood-based matrix that during a four week time period will be remodeled in ECM, mainly through the deposition of 
collagen fibers by activated fibroblast. The model is based upon a schematization that sees the main cells flows among the different regions, moved by the chemokines gradient through the permeability of blood vessels walls for the extravasation and of the blood-based/extracellular matrix for passing across the tissues (Fig. 1). Once arrived in the destination areas, the fibroblast collagen production in region $\mathrm{B}$ and the monocytes/macrophages evolution in FBGC in region $\mathrm{A}$ are modeled.

\section{A. Equation of the model}

According to the block scheme in Fig. 1 the FBR mathematical model can be defined by the dynamic equations of the two main cells in the different regions: monocytes/macrophages and fibroblasts in A, B, C and D.

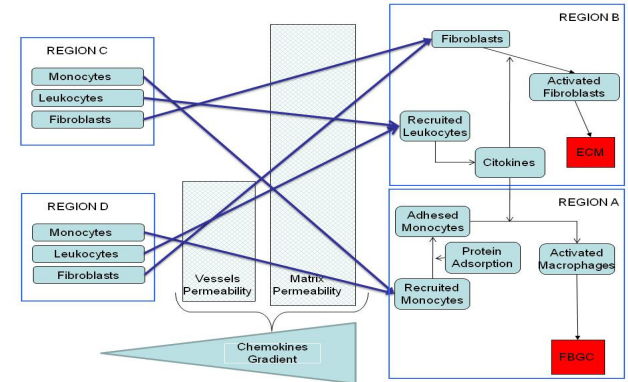

Figure 1: Main blocks scheme of the model. Blue thick arrows represents cell flows

1) Monocytes dynamics and formation of region $A$

The dynamics of monocytes/macrophages in region $\mathrm{B}$ can be defined by the following equation:

$$
\dot{M}^{B}=\dot{M}_{R}{ }^{C}+\dot{M}_{R}{ }^{D}-\dot{M} \dot{M}_{A D H}+\dot{M}^{B}{ }_{B O R N}-\dot{M}^{B}{ }_{\text {DIED }}
$$

where the first three terms are due to cellular flows among different contiguous regions (from region $\mathrm{C}$ and $\mathrm{D}$, and to region A respectively), while the last two are related to the cellular turn-over, with $\dot{M}_{B O R N}^{B}$ modeled by a logistic growth and $\dot{M}_{D I E D}^{B}$ described by a first order dynamics.

The $\dot{M}_{R}^{C}$ models the flow of monocytes recruited from region $\mathrm{C}$ to region $\mathrm{B}$, generated by the chemokines gradient through the blood based matrix. Mathematically, this can be described with a mass transfer equation, having the chemokines gradient $\left(G_{C C L}\right)$ as the motive force which moves the monocytes flow against the resistance of the blood based matrix $\left(R_{M}\right)$ :

$$
\dot{M}_{R}^{C}=\frac{\dot{m}_{R} \cdot G_{C C L}(t)}{R_{M}}
$$

In eq. (2), the term $\dot{m}_{R}^{C}$ is the specific monocytes flow which is proportional to the monocytes concentration in $\mathrm{C}\left(M^{C}\right)$.

Analogously, the flow of monocytes going from region D to region $\mathrm{B}$ can be defined as follows:

$$
\dot{M}_{R}{ }^{D}=\frac{\dot{m}_{R}^{C} \cdot G_{C C L}(t)}{R_{v}+R_{M}}
$$

where $R_{V}$ is the resistance for extravasation through the vessel wall, and $\dot{m}_{R}^{C}$ is proportional to the monocytes concentration in region $\mathrm{D}\left(M^{D}\right)$. The term $\dot{M}_{A D H}$, which represents the monocytes migration from region $\mathrm{B}$ to region $\mathrm{A}$ due to the adhesion with the adsorbed protein upon the electrode coating surface, is modelled as:

$$
\dot{M}_{A D H}=\left(k_{I g G}^{a d h}+k_{F I B R}^{a d h}\right) \cdot M^{B}
$$

where $k_{I g G}^{a d h}$ and $k_{F I B R}^{a d h}$ are the coefficients that represent the capability of induct monocytes adhesion of respectively $\mathrm{IgG}$ and fibrinogen, that are the two main proteins that adsorb upon a foreign material surface in the body; these coefficients depend on the material coating the electrode.

To have a complete description of the system modelled so far, we need to define the time course of some variables used in the previous equations; in particular $G_{C C L}, R_{V}, R_{M}$ have to be mathematically defined. As regards the chemokines gradient $\left(G_{C C L}\right)$, its time variation has been defined using a Gaussian function fitting the experimental values for the chemokine MIP-1 $\beta$ taken from [6]. The resistance for extravasation $R_{V}$ is the inverse of the vascular permeability $\left(P_{V}\right)$, which mainly depends on the mechanical insertion damage $(D)$, and can be expressed by the equation:

$$
P_{V}=\frac{1}{R_{V}}=k_{E V} \cdot f_{P E C}(t) \cdot D
$$

where $f_{P E C}(t)$ is a function of the simulation time, and was chosen according with the time-course trend of the proextravasation cytokines (PEC) concentration during the inflammatory response to the foreign body. This is justified by the fact that PEC are the major factors accountable for the increment of the vascular permeability. In particular $f_{P E C}(t)$, reaches its peak quite immediately after the stimulus (electrode insertion) and then follows an exponential decay during the progression of the inflammatory response. As regards the mechanical insertion damage $(D)$, it depends from the electrode size and shape, and from the insertion angle, defined as the angle between the longitudinal axis of the electrode and that one of the nerve:

$$
D=\frac{\pi}{4} \cdot d_{H}^{2} \cdot \frac{1}{\sin (\theta)}
$$

where $d_{H}$ is the hydraulic diameter of the electrode, defined as 4 times the cross sectional area divided by the perimeter, and $\theta$ is the insertion angle. Similar to the resistance for extravasation $R_{V}$, the resistance of the blood-based matrix $R_{M}$ is the inverse of its permeability $\left(P_{M}\right)$. To properly introduce the definition of this function, it could be useful to recall the main physiological mechanisms underlying the formation of the blood-based matrix. The mechanical damage made by the insertion produces the rupture of blood vessels and an organization clots-like of the extracellular space occupied by a blood-based matrix. During the evolution of FBR the matrix progressively changes toward a fibrosis. This network works as structural scaffold for cells migration through the tissues, thus an optimal dimension of the loops of the net, reflected by an optimal matrix density $\left(\rho_{M}^{*}\right)$, is required for achieving an high matrix specific permeability $\left(p_{M}\right)$. A higher or a lower value of matrix density results in an overall reduction of the matrix permeability $\left(p_{M}\right)$. Thus, matrix specific permeability $\left(p_{M}\right)$ can be described by a nonmonotonic function of matrix density $\left(\rho_{M}\right)$, with a peak value $\left(p_{M}^{\max }\right)$ corresponding to the optimal matrix density $\left(\rho_{M}^{*}\right)$. To this purpose we defined the function $p_{M}=r_{M}^{-1}=f\left(\rho_{M}\right)$ as the polynomial function of third degree. As regards the time course of the matrix density $\left(\rho_{M}\right)$ during the inflammation 
process, it reaches its peak among day 2 and day 8 after the insertion, when it is constituted by platelets and organized fibrin. After the pick $\rho_{M}$ start to decreases because of the cleaving action of the MMPs that attack the fibrin network. It is reasonable to say that wider and heavier is the damage $(D)$ and more important is the activation of surrounding tissue and faster is the organization of the clot that reaches its density peak in few days and viceversa. Given that the is maximal for an optimal $\rho_{M}\left(\rho_{M}^{*}\right)$, that is reached both in the ascending and in the descending part of the $\rho_{M}(t)$ curve, the trend of $p_{M}(t)$, according with this modeling strategy, presents a double-hump shape as showed in the second row of Fig. 2. The value of the pick of the overall matrix permeability $\left(P_{M}\right)$ is further influence by the damage $(D)$, as showed in the last row of Fig 2, because the amount of damage influences the extension of matrix involved in the cellular migration: $\left(P_{M}=p_{m} \cdot h D\right)$. Once we obtain the concentration of adhered monocytes from Eq. (4) the next equation define the concentration of FBGC in region A (expressed as the number of FBGC per surface unit).

$$
F B G C=\left(k_{I g G}^{f u s}+k_{F I B R}^{f u s}\right) \cdot M_{A D H}
$$

where $k_{I g G}^{f u s}$ and $k_{F B B R}^{f u s}$ are the constant of fusion for $M_{A D H}$ toward generate FBGC relative to $\mathrm{IgG}$ and Fibrinogen respectively taken from $[7,8]$ and change during the time of simulation with a sigmoidal shape with plateau $[6,9]$.

FBGC are the main component that constitute Region A of the FBR capsule and in this region are tight packed forming a dense component. Their spatial organization can be geometrically schematize, assuming that the shape of a giant cell is similar to a thin ellipsoid with its major area (axes:

$\alpha_{F B G C}^{m a j}$ and $\left.\alpha_{F B G C}^{\min }\right)$ smashed toward the electrode surface, with the aim of incorporating the foreign body, and the thickness twenty fold smaller than the minor axis $\left(\alpha_{F B G C}^{\text {Thick }}=\frac{1}{20} \alpha_{f B G C}^{\min }\right)$.

Indeed FBGCs in region $\mathrm{A}$ are packed with one of this $\alpha_{F B G C}^{T h i c k}$ axis directed toward the electrode. According to this schematization, the thickness of region $\mathrm{A}$ can be mathematically obtained from the next equation:

$$
\text { Thick }^{A}=\alpha_{F B G C}^{\text {thick }} \cdot L_{F B C G}=\frac{1}{20} \alpha_{F B G C}^{\min } \cdot L_{F B G C}
$$

where $\alpha_{F B G C}^{\min }$ is the minor axis of the ellipsoid representing the FBGC shape and $L_{F B G C}$ is the number of layers made by FBGC around electrode surface. $L_{F B G C}$ depends on FBGC concentration as defined in the following equation:

$$
L_{F B G C}=F B G C \cdot \bar{S}_{F B G C}
$$

where $\bar{S}_{F B G C}$ is the mean value of the area of a FBGC maximal surface along a section plane perpendicular to the longitudinal axis of the electrode, and passing through the major axis of the ellipsoid as is reported in literature [10].

According to the geometrical schematization of FBGC given above, this area corresponds to:

$$
\bar{S}_{F B G C}=\pi \cdot\left(\frac{\alpha_{F B G C}^{m a j}}{2} \cdot \frac{\alpha_{F B G C}^{\min }}{2}\right)
$$

And the minor axis is

$$
\frac{\alpha_{F B G C}^{m a j}}{2}=2 \cdot \alpha_{F B G C}^{\min }
$$

Thus we have that:

$$
\alpha_{F B G C}^{\text {Thick }}=\frac{1}{20} \alpha_{F B G C}^{\min }=\frac{1}{20} \sqrt{\frac{2 \cdot \bar{S}_{F B G C}}{\pi}}
$$

Substituting the eq. (9) and (12) in (8) the thickness of region A is:

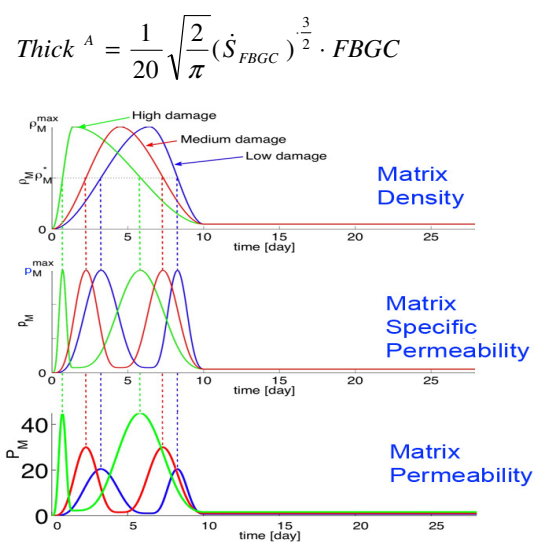

Figure 2: In the upper row the time evolution of the density of the matrix $\left(\rho_{m}\right)$, in the second row the matrix specific permeability $\left(p_{m}\right)$ and in the lower row the matrix permeability $\left(P_{M}\right)$. Different colors correspond to different grade of the damage $(D)$.

2) 8.3.2 Fibroblasts dynamics and formation of region $B$ Fibroblasts dynamics and collagen secretion in region $B$ has been modeled by deriving most of the equations from the work proposed by Dale et colleagues about the collagen formation in dermal wound healing [11]. Main actors of this process are fibroblasts, collagen and the family of its cleaving enzymes called metalloproteinases (MMPs) including the collagenase, and the pro-fibrotic cytokine TGF $\alpha$. Both MMPs and TGF $\alpha$ are produced by fibroblasts and adhered monocytes activated in macrophages histotype.

Fibroblast concentration in region $\mathrm{B}\left(\mathrm{F}^{\mathrm{B}}\right)$ is described by the following equation:

$$
F^{B}=\left(\alpha_{1}+\alpha_{2} T G F\right) F^{B}\left(1-\frac{F^{B}}{k_{c}}\right)-\alpha_{3} F^{B}+\dot{F}_{R}^{C}+\dot{F}_{R}^{D}
$$

$\mathrm{F}^{\mathrm{B}}$ is influenced by a logistic growth term, which represents mitotic generation (first term of eq. (14)). Cell growth is enhanced by TGF $\alpha$, where $\mathrm{a}_{1}$ and $\mathrm{a}_{2}$ are the parameters regulating the growth rate and $\mathrm{k}_{\mathrm{c}}$ is the carrying capacity of the environment. Cells die at a constant rate $\mathrm{a}_{3}$. Similarly as seen for monocytes, fibroblasts are recruited both from contiguous tissues ( $\dot{F}_{R}^{C}$ from region $C$ ) and from the blood ( $\dot{F}_{R}^{D}$ from region D) thanks to a CXCL family chemokines, mainly IL-8, gradient ( $\left.G_{C X C L}\right)$, through the resistance of blood vessels wall $\left(R_{V}\right)$ and of the extracellular matrix $\left(R_{M}\right)$. The equations describing the dynamics of recruited fibroblasts are analogous to those used for monocytes (see eqs. (2) and (3)). Fibroblast proliferation and collagen synthesis are up-regulated by the cytokine called $\mathrm{TGF} \alpha$, as described by the following equation:

$$
T G F=\left(\frac{\alpha_{4} T G F}{1+\alpha_{3} T G F}-\alpha_{6} T G F\right) F+\alpha_{7} M_{A D H}
$$

Concentration of TGF $\alpha$ is governed by an autocrine mechanism in the fibroblasts, described by the first term of Eq. 15. Natural decay of $\mathrm{TGF} \alpha$ is modeled as a first order process with time constant $\mathrm{A}_{6}$ [12]. The last term corresponds to TGF $\alpha$ production by adhered monocytes. 
MMPs dynamics is modeled by the equation:

$$
M M P=\left(\frac{\alpha_{8} F^{B}}{1+\alpha_{9} T G F}-\alpha_{10} M M P\right) F+\alpha_{11} M_{A D H}
$$

MMPs bind to collagen breaking down the fibers. They are secreted by fibroblasts, but the secretion is inhibited by the presence of TGF $\alpha$. Again, the natural decay is taken to be of first order. The last term corresponds to production by adhered monocytes. Collagen concentration depends on the concentration of fibroblasts and TGF $\alpha$, as described by the first term of the following equation. Collagen is degraded by MMPs as described by the last term of the equation.

$$
\dot{C}=\left(\alpha_{12}+\alpha_{13} T G F\right) F^{B}-\alpha_{14} M M P \cdot C
$$

Where, $\mathrm{a}_{12} \mathrm{~F}^{\mathrm{B}}$ is the basal production of collagen; $\mathrm{a}_{13} \mathrm{TGF}^{\mathrm{B}}$ is the amount of collagen production induced by TGF $\alpha$.

Assuming that the typical average concentration of collagen in region $\mathrm{B}\left(\bar{C}_{B}\right)$ is known, it is possible to estimate the superficial concentration of collagen as the product of $\bar{C}_{B}$ by the thickness of region $\mathrm{B}\left(\right.$ Thick $\left.^{B}\right)$. At the same time, this superficial concentration has to be equal to the time integral of the superficial production of collagen, expressed by the product of collagen production $(\dot{C})$ by Thick $^{B}$.

Thus, the following equation is able to describe the relation between $\dot{C}$ and Thick $^{B}$ :

$$
\int_{t_{0}}^{t} \dot{C} \cdot \text { Thick }^{B} d t=\bar{C}_{B} \cdot \text { Thick }^{B}
$$

By differentiation both the terms of eq. (18), Thick $^{\mathrm{B}}$ is expressed in function of $\dot{C}$ :

$$
\begin{gathered}
\text { Thick }^{B}=\frac{\text { CThick }^{B}}{\bar{C}_{B}} \\
\text { Thick }^{\text {FBR }}=\text { Thick }^{A}+\text { Thick }^{B}
\end{gathered}
$$

\section{RESULTS}

For this preliminary simulation of the model has been considered an electrode with its geometrical properties resembling the tf-LIFE4s, but implanted perpendicularly to the nerve. Adhesion and fusion percentage of monocytes and chemokines gradients have been gathered from data reported by $[6,9]$ for polyethylene terephthalate (PET), that presents the same water contact angle than polyimide (about $70^{\circ}$ ).

The model has been implemented in MATLAB/Simulink using ODE45 solver for the differential equations.

In Fig. 3 the time course of the number of FBGCs for square millimeter, the number of fibroblasts in region $\mathrm{B}$ for cubic millimeter, the thickness of region A, B and the total thickness of the capsule are represented. In agreement with the fact that the reaction became stable after about 4 weeks, values tend to stabilize when overcome day 25 .

\section{DISCUSSION AND CONCLUSIONS}

Aim of this model is to gather an idea of the influence of geometrical and chemical parameters that characterize an electrode upon the foreign body reaction produced by the implantation of the electrode in a peripheral nerve. This tool is useful even before that the electrode is fabricated and tested, thus allowing electrode developers to spare time and economic resources and to evaluate possible hypothetical solutions for accounting the tissue-electrode mismatch. The dynamics of the processes have been modeled starting from a deep analysis of the literature about FBR, in particular in the nervous system. The parameters have been taken from published articles, favoring especially the data regarding the PNS, or have been settled according with the most plausible results of simulations. The output of the simulation with an electrode that resemble the characteristic of tfLIFE is consistent with the histological description reported by Lago et colleagues [13]. Nevertheless the model will profit from a experimental validation in vitro and in vivo, adopting different materials as foreign body or different coating surfaces. Because biocompatibility is a functional characteristic, which requires an analysis of tissue response using an electrically functional implant, the future version of the model should take into account also the electrical properties of a stimulating/recording working electrode.

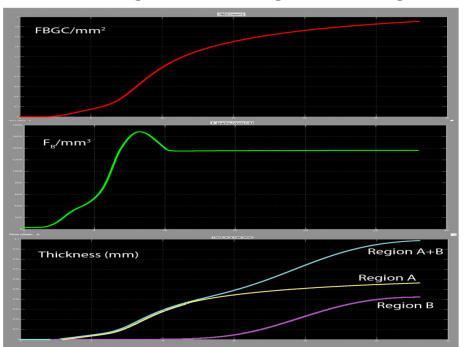

Figure 3: Time course of the number of FBGCs for square millimeter, number of fibroblasts in region $B$ for cubic millimeter and the thickness of region A, B and the total thickness of the capsule.

\section{REFERENCES}

[1] Biran R, Martin DC, Tresco PA. Neuronal cell loss accompanies the brain tissue response to chronically implanted silicon microelectrode arrays. Exp Neurol. 2005 Sep;195(1):115-26.

[2] Schwartz AB, Cui XT, Weber DJ, Moran DW. Brain-controlled interfaces: movement restoration with neural prosthetics. Neuron. 2006 Oct 5;52(1):205-20.

[3] Vodovotz Y, Constantine G, Rubin J, Csete M, Voit EO, An G. Mechanistic simulations of inflammation: current state and future prospects. Math Biosci. 2009 Jan;217(1):1-10.

[4] Nichols MF, Greller LD, Hahn AW. A mathematical model of the foreign body reaction to implanted materials. Biomed Sci Instrum. 1979 Apr 23-25;15:1-5.

[5] Su J, Perez-Gonzalez H, Tang L. Modeling and simulation of foreign body reactions to neural implants; 2007.

[6] Jones JA, Chang DT, Meyerson H, Colton E, Kwon IK, Matsuda T, et al. Proteomic analysis and quantification of cytokines and chemokines from biomaterial surface-adherent macrophages and foreign body giant cells. J Biomed Mater Res A. 2007 Dec 1;83(3):585-96.

[7] Shen M, Garcia I, Maier RV, Horbett TA. Effects of adsorbed proteins and surface chemistry on foreign body giant cell formation, tumor necrosis factor alpha release and procoagulant activity of monocytes. J Biomed Mater Res A. 2004 Sep 15;70(4):533-41.

[8] Shen M, Horbett TA. The effects of surface chemistry and adsorbed proteins on monocyte/macrophage adhesion to chemically modified polystyrene surfaces. J Biomed Mater Res. 2001 Dec 5;57(3):336-45.

[9] Rodriguez A, Meyerson H, Anderson JM. Quantitative in vivo cytokine analysis at synthetic biomaterial implant sites. J Biomed Mater Res. A. 2009 Apr;89(1):152-9.

[10] Anderson JM. Multinucleated giant cells. Curr Opin Hematol. 2000. 7:40-47.

[11] Dale PD, Sherratt JA, Maini PK. A mathematical model for collagen fibre formation during foetal and adult dermal wound healing. Proc Biol Sci. 1996 May 22;263(1370):653-60.

[12] Sporn MB, Roberts AB. TGF-beta: problems and prospects. Cell Regul. 1990 Nov;1(12):875-82.

[13] Lago N, Yoshida K, Koch KP, Navarro X. Assessment of biocompatibility of chronically implanted polyimide and platinum intrafascicular electrodes. IEEE Trans Biomed Eng. 2007 Feb;54(2):281-90. 